\title{
Study on the effect of nano-hydroxyapatite on bone repair of athletes
}

\author{
Jianyong Wang \\ College of Physical Education, Zhengzhou Normal University, Zhengzhou, China
}

Videosurgery Miniinv 2018; 13 (2): 221-226

DOI: https://doi.org/10.5114/wiitm.2018.73973

\begin{abstract}
Introduction: Minimally invasive surgery is a widely used method for bone repair. Nano-hydroxyapatite ( $n$-HA) is a non-toxic and harmless bone repair material.

Aim: To study the repair effect of n-HA/PA66 on the lumbar vertebrae injury of basketball players.

Material and methods: Firstly, the n-HA/PA66 material was prepared and its properties were studied. Then, the $n$-HA/PA66 material was used in a rabbit vertebral replacement experiment to study its bone coverage, fiber coverage and bone density. Afterwards, the material was used in vertebral injury treatment of basketball players to study its bone repairing effect.

Results: It was found that the fiber coverage of artificial vertebral bodies of n-HA/PA66 material tended to decrease with the increase of replacement time but no significant difference was found in the fiber coverage at different times ( $p>0.05)$; the longer the replacement time, the greater the bone coverage, with statistically significant differences ( $p<0.05)$; as the replacement time increased, the density and osteogenic volume increased; the Japanese Orthopaedic Association (JOA) scoring both 3 months and 6 months after surgery was greater than that immediately after surgery, with a significant difference ( $p<0.05)$; the difference of angle of Cobb showed a decreasing trend with the increase of replacement time, and there was a significant difference ( $p<0.05)$; follow-up results showed that the subjects had no rejection reaction.

Conclusions: N-HA material played an important role in basketball players' bone repair treatment and is worth being promoted for application.
\end{abstract}

Key words: nano-hydroxyapatite, bone repair, polyamide.

\section{Introduction}

Basketball is a hand-centered body antagonistic movement, with confrontational and collective characteristics, which requires players to run fast, keep jumping and compete with each other in strength [1]. As a result, injuries are inevitable during the exercise, among which vertebral injury occurs frequently, mainly due to hitting the knee or elbow or inappropriate landing after jumping [2]. For this injury, bone repair technology is generally applied.
Sella et al. [3] studied the bone injury of the femur in mice and found through low intensity laser therapy that inflammatory infiltration, bone trabeculae and periosteal formation were significantly reduced while new bone was increased, indicating that low intensity laser therapy had a good effect on bone injury. For spinal cord injury, Cox et al. [4] through preclinical studies and pharmacological therapies investigated the mechanism of homologous hypoxia and ischemia, excitotoxicity, inflammation, apoptosis, epigenetic changes, clinical trials and myelin 
regeneration. Nagoshi et al. [5] identified the mechanism of spinal cord injury, focusing on the effects of riluzole, minocycline, Rho protein antagonist, polyethylene glycol prepared magnesium chloride and basic fibroblast growth factor. The most ideal repair materials for bone injuries are those with good tissue compatibility, bone conduction and induction ability and biodegradability as well as high safety and diverse sources [6]. Nano-hydroxyapatite ( $n-\mathrm{HA})$ is an important component of bones and teeth of vertebrates and has good bone conductivity. The molecular structure of nano-hydroxyapatite and its proportion of calcium and phosphorus are similar to those of natural bone, and it is non-toxic and non-irritating [7, 8] and is therefore widely applied in bone tissue repair. Combining n-HA with polyamide 66 (PA-66), this study investigated the repair of vertebral injury of basketball players so as to provide some reference for the bone repair of basketball players.

\section{Aim}

This study aims to investigate the role of $n-H A$ combined with PA-66 in the bone repair of athletes under minimally invasive surgical treatment and provide some reference for bone repair treatment and actively promote the scientific implementation of bone repair.

\section{Material and methods}

\section{Experimental objects}

Four New Zealand white rabbits aged 7 weeks with a weight of $3.41 \pm 0.4 \mathrm{~kg}$ were selected from the Experimental Animal Center of Zhengzhou University. The experiment was completed in the Chemistry Experimental Center of Zhengzhou Normal University, strictly following the relevant feeding standards. Additionally, 10 basketball players with vertebral injury accepted by Zhengzhou Central Hospital during January 2017 to July 2017 were included. All the subjects and their families were informed of the experimental procedures and signed the informed consent.

\section{Materials and equipment}

The materials and equipment included are as follows: DMEM (Suolaibao Technology Co., Ltd. China), fetal bovine serum (Beijing Yuhengfeng Technolo- gy Co., Ltd. China), dimethyl sulfoxide (Changzhou Zidong Lianhua Import and Export Co., Ltd. China), pentobarbital (Beijing Pubosi Bio Co., Ltd. China), PA66, (Dongguan Yisike Plastic Co., Ltd. China), diammonium phosphate (Suzhou Youhesi Chemical Technology Co., Ltd. China), constant temperature incubator (Dongguan Weihuang Test Equipment Co., Ltd. China), microplate reader (Shanghai Shanpu Biotechnology Co., Ltd. China), cell incubator (Shanghai Zhichu Instrument Co., Ltd. China), inverted microscope (Shanghai Caikang Optical Instrument Co., Ltd. China), centrifuge (Beijing Shidai Beili Centrifuge Co., Ltd. China), distracter (Jiangsu Yunzhong Medical Technology Co., Ltd. China).

\section{Experimental method}

\section{Preparation of n-HA/PA66 materials}

(1) $100 \mathrm{ml}$ of $0.2 \mathrm{~mol} / \mathrm{l}$ aqueous solution of diammonium hydrogen phosphate was added dropwise to $0.4 \mathrm{~mol} / \mathrm{l}$ calcium nitrate solution of the same volume, and mixed with the original solution by stirring at $400 \mathrm{rpm}$. Then, the obtained solution was heated by water bath at $30^{\circ} \mathrm{C} / \mathrm{h}$, with the $\mathrm{PH}$ value maintained at 10 .

(2) Afterwards, the solution was placed at room temperature for sedimentation and centrifuged at $600 \mathrm{rpm}$ for $10 \mathrm{~min}$, after which the precipitate was collected and cleaned with ionized water for $10 \mathrm{~min}$. Then, the collected nanoparticles were placed at $-50^{\circ} \mathrm{C}$ for freezing to obtain $\mathrm{n}-\mathrm{HA}$ particles.

(3) The n-HA and PA66 materials were added to anhydrous ethanol with blowing agent and mixed by stirring.

(4) The mixed material was then placed in an $85^{\circ} \mathrm{C}$ vacuum oven for heating and drying until the ethanol was fully evaporated.

(5) Dry ingredients of different quality were weighed and added to a $5 \mathrm{~cm} \times 3 \mathrm{~cm} \times 1 \mathrm{~cm}$ grinding apparatus which was placed on a vulcanizer, with the pressure set at $5 \mathrm{MPa}$ and the temperature at $300^{\circ} \mathrm{C}$. After $60 \mathrm{~min}$ at constant temperature, they were cooled to room temperature.

(6) The obtained material was cleaned and dried.

\section{Rabbit prosthesis transplantation experiment}

After the rabbits were anesthetized with pentobarbital at a concentration of 3\%, an oblique notch was cut at the cervical anterior of each rabbit. Re- 
placement was performed with n-HA/PA66 artificial lumbar vertebrae and location adjustment was carried out under X-ray, followed by sealed suture. After the operation, the behavior of the rabbits was not restricted. Two, four, eight and sixteen weeks after the lumbar vertebrae replacement, one rabbit was selected and put to death to carry out X-ray examination of lumbar vertebrae in rabbits. Specifically, the rabbits' n-HA/PA66 artificial lumbar vertebrae and surrounding tissues were extracted and sliced with the non-decalcified hard tissue slicing method to observe the fiber coverage, bone coverage and bone density under an inverted microscope.

\section{Minimally invasive treatment of patients}

The 10 basketball players were placed on transparent beds and anesthetized with endotracheal intubation. Operative segmental positioning was performed and the skin incision was marked and zoomed step by step. Additionally, the MAST Quadrant channel was placed, which initially expanded vertically along the muscle and subsequently adjusted the angle to reveal the articular process sufficiently that it formed an angle of $15^{\circ}$ with the sagittal plane. The free arm was locked, the MAST Quadrant channel was fixed, the cold light source was connected and intervertebral foramen decompression under direct vision was carried out. Patients with central spinal stenosis could reduce the pressure through high-speed drilling. The intervertebral space was cleaned and the cartilage end plate was scraped until punctate bleeding appeared. An intervertebral distractor was used to distract the disc space to a reasonable height. According to the volume of the patient's bone trough, the n-HA/PA66 material artificial vertebral body with appropriate size and length was selected. Afterwards, the cut bone was crushed into the bone groove and fixed with a steel plate. The pedicle screw and connecting rod were placed with the apex of the triangular fossa positioning and the MAST Quadrant channel was removed. The study time was immediately after surgery, 3 months after surgery and 6 months after surgery and the indexes include JOA score and Cobb angle difference.

\section{JOA scoring standard}

The JOA score reflects the extent of human functional disorder and its scoring covers three aspects which are upper limb, lower limb and feeling, with each standard as follows:

Upper limb scoring: 0: unable to hold chopsticks or spoons; 1 : able to hold spoons; 2 : able to hold chopsticks; 3: able to hold chopsticks and do simple housework; 4: able to do normal activities.

Lower limb scoring: 0: unable to walk; 1 : able to walk with assistance; 2: able to climb steps with support; 3: able to walk without support but inflexible; 4: able to walk normally.

Feeling scoring: 0: with obvious sensory disturbances; 1: with slight sensory disorder or numbness; 2: with no sensory disorder [9].

\section{VAS grading standards}

A $10 \mathrm{~cm}$ horizontal line was drawn on a piece of paper. The left end marked the number 0 , indicating no pain, and the right end marked the number 10 , indicating a strong feeling of pain. The middle section indicated different degrees of pain. The patients were asked to mark on the line according to their own pain feeling. The mean value of severe pain was $8.41 \pm 1.35$, the mean value of average pain was 5.18 \pm 1.41 , and the mean value of mild pain was 2.57 \pm 1.04 [10].

\section{Statistical analysis}

The statistical analysis of the data were completed using SPSS 18.0. When the data were normally distributed, single factor variance analysis was adopted. The differences were considered statistically significant when $p<0.05$.

\section{Results}

\section{Basic information of the players}

The basketball players had been engaged in a basketball career for 5 to 7 years, with an average age of $25.4 \pm 2.6$ years, an average weight of 74.23 $\pm 2.11 \mathrm{~kg}$ and an average height of $1.86 \pm 3.64 \mathrm{~m}$, with no other bone injuries. There was no significant difference between the above indexes $(p>0.05)$. The vertebral injury of the players is shown in Photo 1.

\section{Preparation of N-HA/PA66 material}

As shown in Photo 2, the n-HA/PA66 material has evenly distributed holes on its surface, which connects to tubular channels, with light quality and strong plasticity. 


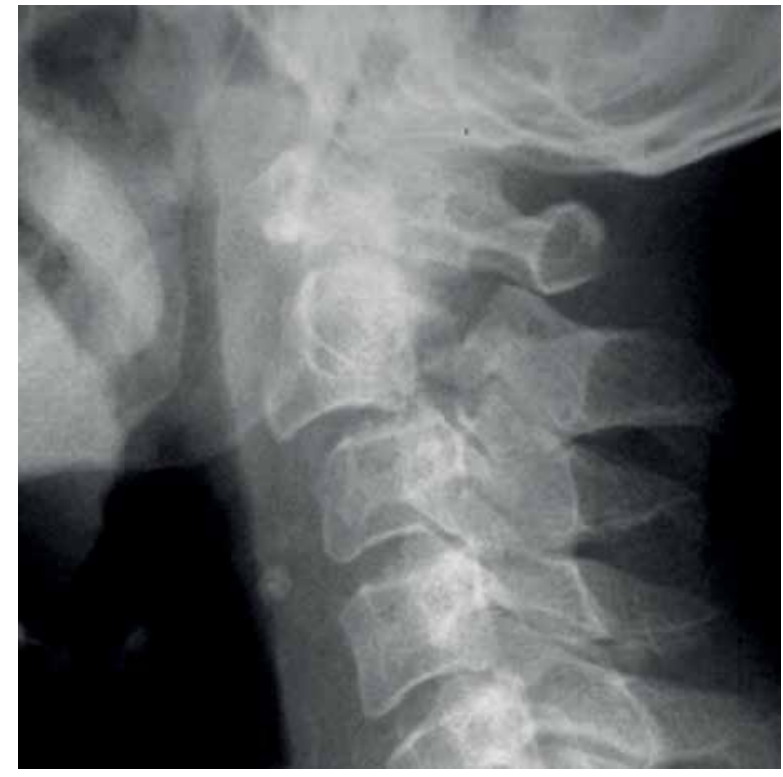

Photo 1. Bone injury

\section{VAS scoring of minimally invasive treatment}

The VAS score of the patients before surgery was $6.9 \pm 0.7$ and the situation was improved 2 weeks after surgery, i.e., it dropped to $3.2 \pm 0.7$. Four weeks and 8 weeks after surgery, it was $2.6 \pm 0.4$ and 1.1 \pm 0.3 . Sixteen weeks after surgery, it was $0.3 \pm 0.1$, indicating that the pain gradually disappeared 16 weeks after surgery.

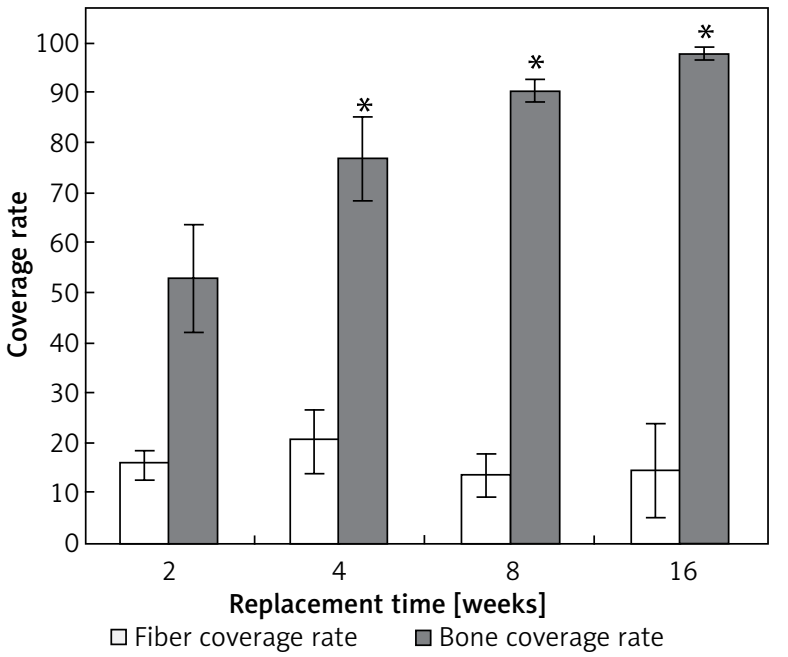

Figure 1. Bone coverage rate of n-HA/PA66 artificial vertebral body at different replacement times

Note: ${ }^{*}$ indicates $p<0.05$ compared to the bone coverage rate at 2 weeks, with significant differences.

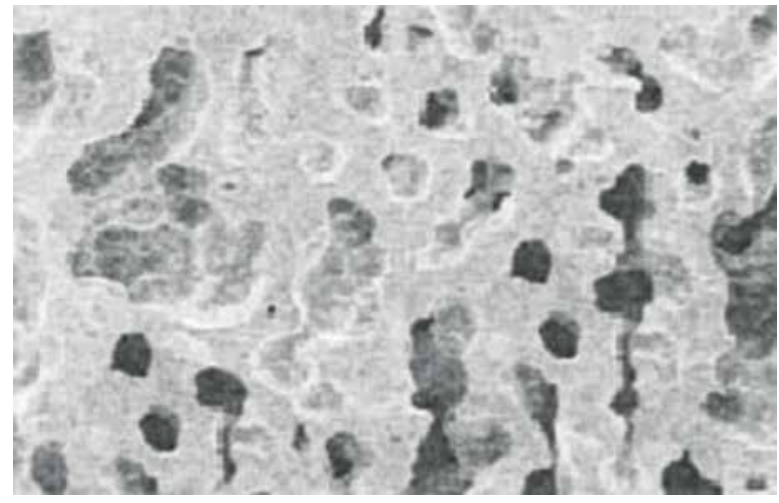

Photo 2. Morphology of n-HA/PA66 material under electron microscope $(1 \mathrm{~mm})$

\section{Fiber coverage and bone coverage}

As shown in Figure 1, the fiber coverage of the n-HA/PA66 artificial vertebral body decreased with the increase of replacement time, but there was no significant difference in fiber coverage at different times $(p>0.05)$. As for the bone coverage, it ranked as 2 weeks $<4$ weeks $<8$ weeks $<16$ weeks. There are significant differences between the bone coverage at 2 weeks and that at the other three times $(p<0.05)$. According to X-ray observations, bone cortical thinning or other undesirable phenomena did not occur in any periods, nor was the artificial vertebral body broken or its surrounding cortex with fuzzy interface.

\section{Changes of bone density}

As shown in Figure 2, the bone density showed a rising trend with the increase of time, with significant differences $(p<0.05)$, suggesting that the amount of osteogenesis increased in the rabbits.

The score of the artificial vertebral body at various times

As shown in Figure 3, the JOA score 3 months and 6 months after surgery was higher than that immediately after surgery, with significant differences $(p<0.05)$. Also, the score between the first two showed no significant differences $(p>0.05)$.

Cobb angle difference of the artificial vertebral body at various times

As shown in Figure 4, the Cobb angle difference decreased with the increase of replacement time and there were significant differences in the angle difference between the three periods $(p<0.05)$. 


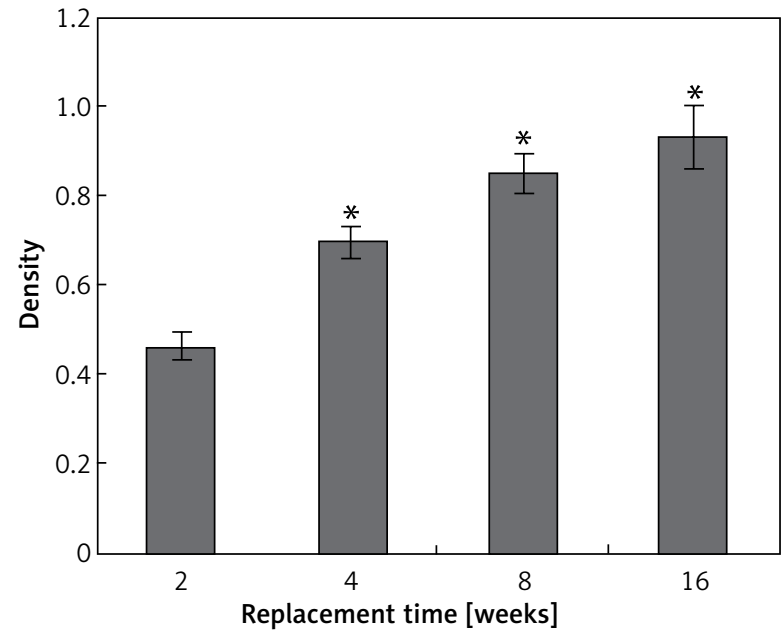

Figure 2. Bone density of n-HA/PA66 artificial vertebral body at different replacement times

Note: ${ }^{*}$ indicates that $p<0.05$ compared to the bone density at 2 weeks, with significant differences.

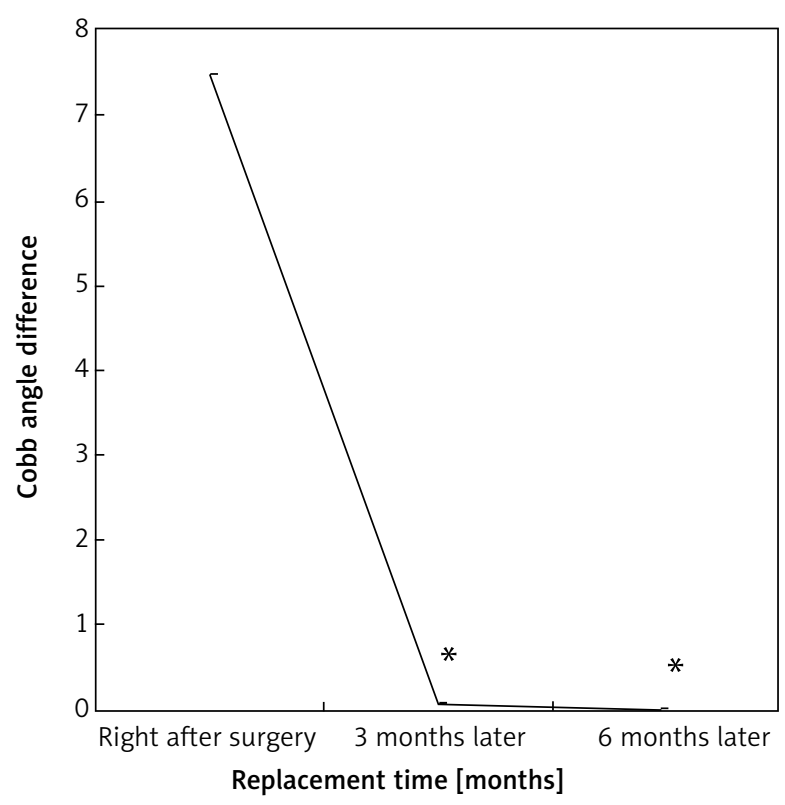

Figure 4. Cobb angle difference of the artificial vertebral body

Note: *indicates that $p<0.05$ compared to that after surgery, with significant differences.

\section{Follow-up results}

After the surgery, the players' physical comfortableness was inquired about. No abnormity was found in the blood test 3 months and 6 months after surgery. Also, the postoperative healing effect was good, with no rejection reaction.

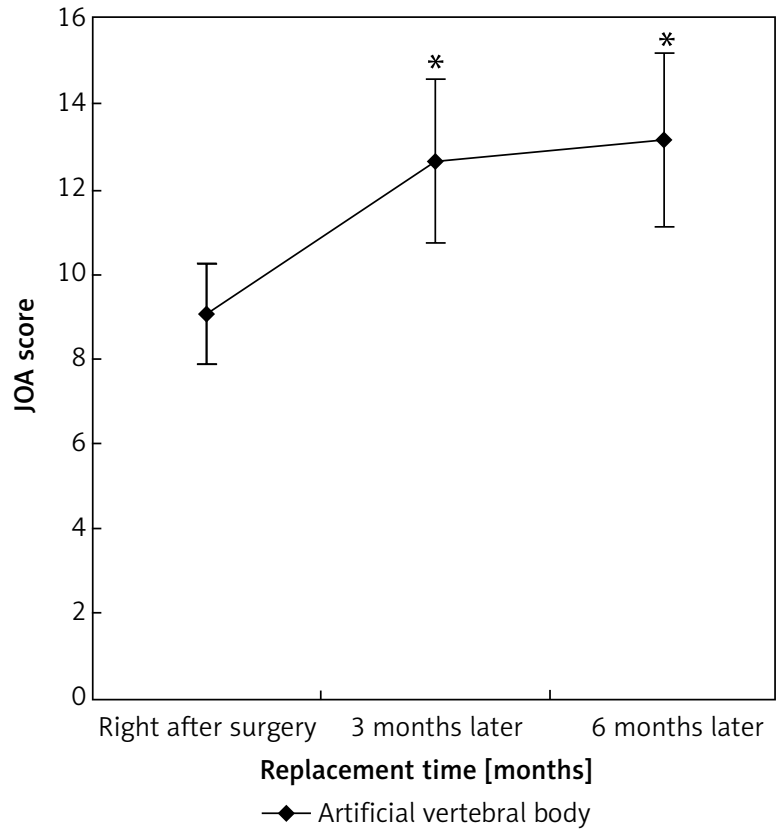

Figure 3. JOA score of the artificial vertebral body

Note: *indicates that $p<0.05$ compared to the score after surgery, with significant differences.

\section{Discussion}

As more and more people participate in the sport of basketball, the cases of bone injury increase. N-HA materials have been used in bone repair therapy recently. Sultana [11] combined nano-hydroxyapatite with polycaprolactone and produced tissue-engineered scaffolds. Dhivya et al. [12] used nano-hydroxyapatite, chitosan and $\beta$-phosphate glycerol to make hydrogel as an alternative to bone loss. With the progress of materials, tissue engineering and other related disciplines, n-HA/PA66 material has obtained good research results in bone repair [13]. In this paper, $\mathrm{n}-\mathrm{HA}$ material and PA66 were combined to make n-HA/PA66 material. The results showed that there was no significant difference in fiber coverage with the increase of time while bone coverage and bone density increased, with significant differences $(p<0.05)$, suggesting that the artificial vertebral body made of $n$-HA/PA66 material had a good effect. Also, the VAS score decreased with the increase of time after surgery while the JOA score increased with the increase of time but with a gradually decreasing degree. At the same time, the Cobb angle difference decreased with the increase of replacement time. According to a study, the Cobb angle can assess the 
bending angle of the vertebral body, reflecting the degree of compression of the vertebral body [14]. In addition, the follow-up results showed that there was no rejection reaction in the players.

\section{Conclusions}

n-HA material combined with minimally invasive surgery had a good effect on the bone repair of athletes and could provide some reference for the treatment of bone repair, which is worth being popularized.

\section{Conflict of interest}

The author declares no conflict of interest.

\section{References}

1. Klupiński K, Krekora K, Woldańska-Okońska M. Evaluation of early physiotherapy in patients after surgical treatment of cruciate ligament injury by bone-tendon-bone method. Pol Merkur Lekarski 2014; 36: 22-7.

2. Osbahr DC, Swaminathan SS, Allen AA, et al. Combined flexor-pronator mass and ulnar collateral ligament injuries in the elbows of older baseball players. Am I Sport Med 2010; 38: 733-9.

3. Sella VRG, Bomfim F, Carolina P, et al. Effect of low-level laser therapy on bone repair: a randomized controlled experimental study. Laser Med Sci 2015; 30: 1061-8.

4. Cox A, Varma A, Banik N. Recent advances in the pharmacologic treatment of spinal cord injury. Metab Brain Dis 2015; 30: 473-82.

5. Nagoshi N, Fehlings MG. Investigational drugs for the treatment of spinal cord injury: review of preclinical studies and evaluation of clinical trials from Phase I to II. Expert Opin Inv Drug 2015; 24: 645-58.

6. Xu N, Ye XJ, Wei DX, et al. 3D Artificial bones for bone repair prepared by computed tomography-guided fused deposition modeling for bone repair. Acs Appl Mater Inter 2014; 6: 14952-63.

7. Ramesh N, Moratti SC, Dias GJ. Hydroxyapatite-polymer biocomposites for bone regeneration: a review of current trends. J Biomed Mater Res B Appl Biomater 2017; (5 Suppl).

8. Macmillan AK, Lamberti FV, Moulton J, et al. Similar healthy osteoclast and osteoblast activity on nanocrystalline hydroxyapatite and nanoparticles of tri-calcium phosphate compared to natural bone. Int I Nanomed 2014; 9: 5627-37.

9. Kato S, Oshima Y, Oka H, et al. Comparison of the Japanese Orthopaedic Association (JOA) score and modified JOA (mJOA) score for the assessment of cervical myelopathy: a multicenter observational study. Plos One 2015; 10: e0123022.

10. Knop C, Oeser M, Bastian L, et al. Development and validation of the Visual Analogue Scale (VAS) Spine Score. Unfallchirurg 2001; 104: 488-97.

11. Sultana N. Fabrication of nanohydroxyapatite/poly(caprolactone) composite microfibers using electrospinning technique for tissue engineering applications. J Nanomater 2014; 2014: 65.
12. Dhivya S, Saravanan S, Sastry TP, Selvamurugan N. Nanohydroxyapatite-reinforced chitosan composite hydrogel for bone tissue repair in vitro and in vivo. J Nanobiotech 2015; 13: 40.

13. Puppi D, Federica C, Piras AM, et al. Polymeric materials for bone and cartilage repair. Prog Polym Sci 2010; 35: 403-40.

14. Yang CW, Li YM, Zhao YF, et al. Adult degenerative scoliosis: can Cobb angle on a supine posteroanterior radiograph be used to predict the Cobb angle in a standing position? Medicine 2016; 95: e2732.

Received: 14.11.2017, accepted: 21.01.2018. 